\title{
Heat Load Forecasting of District Heating System Based on Numerical Weather Prediction Model
}

\author{
YANG Hongying ${ }^{1,}$, JIN Shuanglong $^{1}$, FENG Shuanglei ${ }^{1}$, WANG bo ${ }^{1}$, ZHANG \\ fei $^{1}$, CHE Jianfeng ${ }^{1}$ \\ ${ }^{1}$ China Electric Power Research Institute, Beijing 100192, P.R. China \\ ayanghongying@epri.sgcc.com.cn
}

\begin{abstract}
Keywords: Heating Load Forecast; Numerical Weather Prediction; Artificial Neural Network; Temperature; Weather Forecast.

Abstract. This paper reports an application of Numerical Weather Prediction (NWP) in the heat load forecasting field. The NWP is applied to obtain the correlated weather parameters of the heat load, and then the properly structured Artificial Neural Network (ANN) model is designed to perform the prediction. Satisfactory experimental results are obtained by using actual heat load data in the north of China. The experimental result shows that the proposed NWP based method can predict the heat load precisely. Comparing with the traditional weather forecast based-method, the proposed method can effectively improve the forecasting accuracy.
\end{abstract}

\section{Introduction}

Heat load forecasting plays an important role in the safe and economic operation of the urban district heating system. Accurate heat load forecast provides system dispatchers with timely information to operate the system economically and reliably [1]. Lately, the share of renewable energy in the electricity generation is increasing dramatically [2]. In order to accommodate more renewable energy, the need for accurately forecasting heat load becomes more urgent, due to the restriction between heat and electricity demand in China.

Nowadays a great many of different technologies [3,4] have been applied for heat load forecast, such as Linear Regression [5], ARMA Model [6], Kalman filter [7], grey model [8], Artificial Neural Network [9-11], support vector machine [12]. Many of them have obtained considerable results. However, the heat load is mainly affected by the outdoor environmental parameters which include outdoor temperature, solar radiation, relative humidity, wind speed, pressure and so on [13]. The traditional weather forecast which is used by these methods is not sufficient for the heat load predicting requirements, because the resolution and parameters that the traditional weather forecast provided are quite limited.

In order to solve the problem and further improve the accuracy of the heat load prediction, this paper introduces the Numerical Weather Prediction (NWP) for the forecasting of heat load. After extracting the valid weather parameters which influence the heat load from the NWP, the formulated Artificial Neural Network (ANN) model are employed to forecast the heat load. The proposed method was applied to short-term heat load forecasting and the experimental results based on real data from the north china illustrated the validity of the proposed method.

\section{The Numerical Weather Prediction}

\subsection{Basis theory}

The NWP uses mathematical models of the atmosphere and oceans to predict the weather based on current weather conditions [14]. Though first attempted in the 1920s, it was not until the advent of computer simulation in the 1950 s that numerical weather predictions produced realistic results. Nowadays, a number of global and regional forecast models are run in different countries worldwide, using current weather observations relayed from radiosondes or weather satellites as inputs to the models [15]. 
In this paper, the Multi-Scheme Ensemble Prediction System (MSEPS) produced by WEPROG (Weather and wind Energy PROGnosis) are introduced for the heat load forecasting. MSEPS has been operational since 2004. Based on WEPROGs own NWP formulation, the system is built up with three different dynamics schemes, five different condensation schemes and five different vertical diffusion schemes, which result in an ensemble of 75 members [15]. The characteristic of the MSEPS system is that it has the capabilities to develop physical uncertainties with well-defined differences among the ensemble members. MSEPS system contains a 2-step power prediction module. In the first step a physical reference power is computed and in a second step, the reference power is localized statistically and with the help of weather classes defined by the ensemble weather input [16].

\subsection{The Output Weather Parameters of NWP}

The output weather parameters of the NWP by WEPROG contain the wind speed, wind direction, momentum flux, mean sea level pressure, fraction of clouds, sensible heat flux, latent heat flux, shortwave radiation, longwave radiation, surface pressure, large scale precipitation, convective scale precipitation, temperature, and humidity. The time resolution is 15 minutes. Figure 1 shows the difference of temperature factor between traditional weather prediction and NWP. In order to figure out relationship between the weather parameters and the heat load, the correlation coefficients is employed in the paper to assess the output weather parameters.

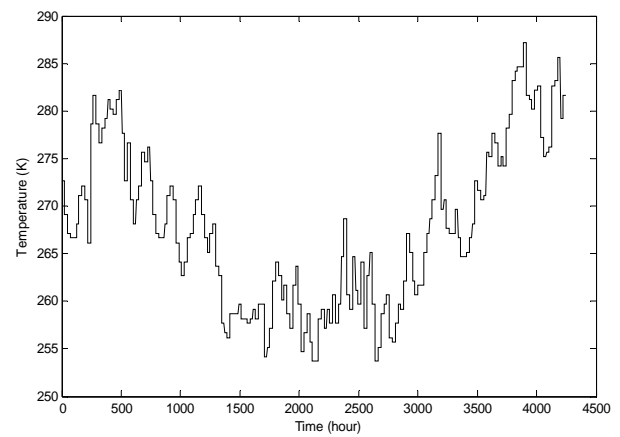

(a) Traditional weather forecast

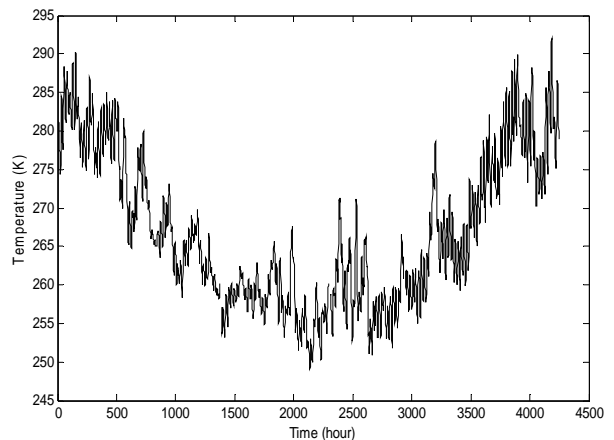

(b) NWP

Fig.1 Comparison between traditional weather forecast and NWP

\subsection{The Impact Factors of Heat load forecasting}

In this study, the heat load data of certain province in the north of China from October 2013 to January 2014 were used to calculate the correlation coefficients. Figure 2 shows the correlation of the temperature and heat load. The correlation coefficient is 0.8670 . This strong correlation indicated that the temperature of NWP should be used as the essential factor in heat load forecasting.

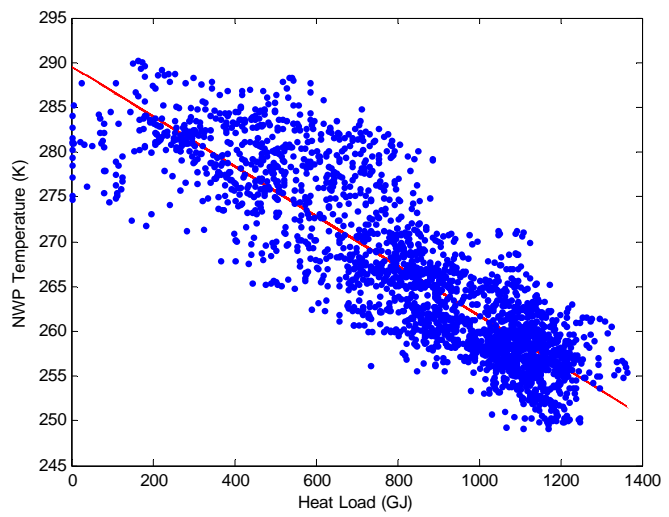

Fig.2 Correlation coefficients between temperature and heat load

Figure 3 partly shows the correlations between the weather parameters and heat load. Table 1 gives the values of the correlation coefficients for all the weather parameters of NWP. Form the figure and table we can see, except temperature parameter, the correlations of longwave radiation and humidity is relatively higher than others. For part of weather parameters, the correlations are almost 0 , which means these parameters have no correlation with the heat load. Therefore, the main impact factors of heat load are temperature, longwave radiation and humidity. 


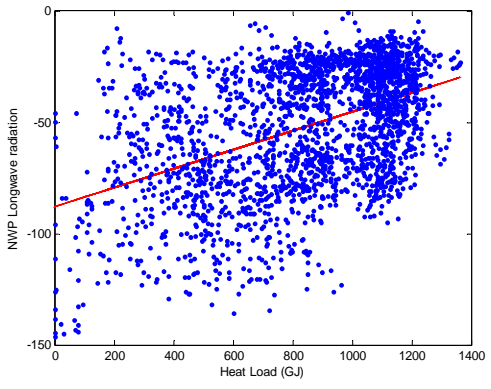

(a) Longwave radiation

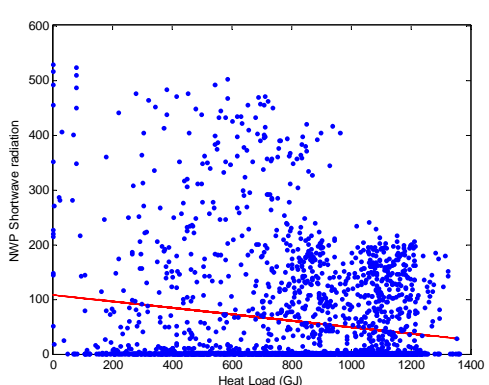

(d) shortwave radiation

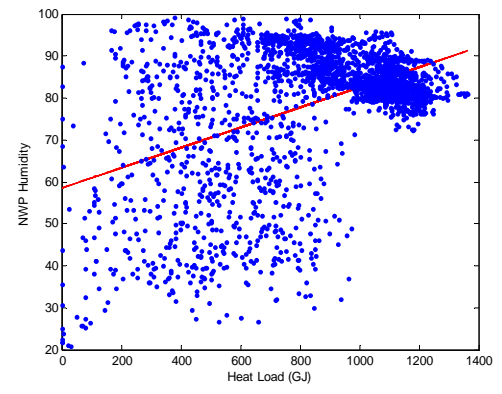

(b) Humidity

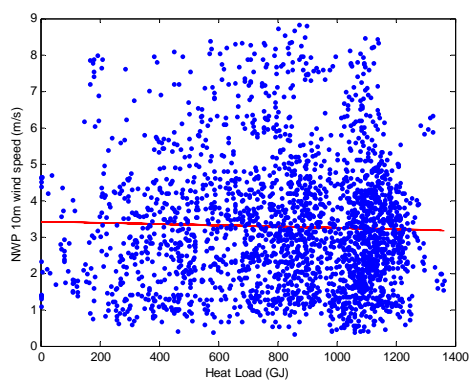

(e) $10 \mathrm{~m}$ wind speed

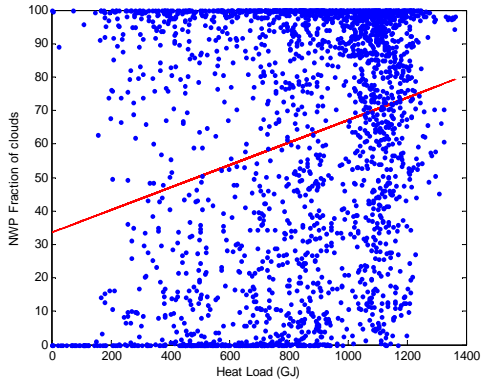

(c) fraction of clouds

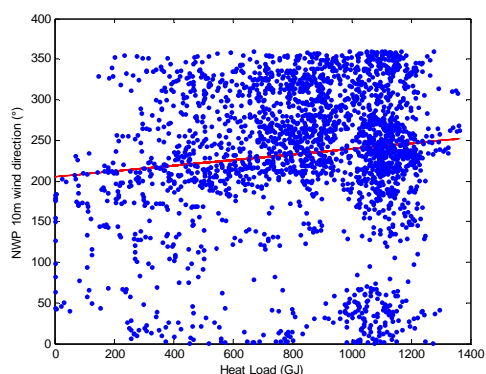

(f) $10 \mathrm{~m}$ wind direction

Fig. 3 Correlation coefficients between part of weather parameters and heat load

Table 1 Correlation coefficients between weather parameters and heat load

\begin{tabular}{cccc}
\hline Weather parameters & $\begin{array}{c}\text { Correlation } \\
\text { coefficients }\end{array}$ & Weather parameters & $\begin{array}{c}\text { Correlation } \\
\text { coefficients }\end{array}$ \\
\hline temperature & $\mathbf{0 . 8 7 6 0}$ & momentum flux & 0.0391 \\
\hline 10m wind speed & 0.0298 & mean sea level pressure & 0.2794 \\
\hline 10m wind direction & 0.1169 & fraction of clouds & 0.2651 \\
\hline 30m wind speed & 0.0356 & sensible heat flux & 0.1145 \\
\hline 30m wind direction & 0.0995 & latent heat flux & 0.1199 \\
\hline $100 \mathrm{~m}$ wind speed & 0.0737 & shortwave radiation & 0.1744 \\
\hline $100 \mathrm{~m}$ wind direction & 0.0967 & longwave radiation & $\mathbf{0 . 4 5 4 4}$ \\
\hline $170 \mathrm{~m}$ wind speed & 0.0282 & surface pressure & 0.1777 \\
\hline $170 \mathrm{~m}$ wind direction & 0.0851 & humidity & $\mathbf{0 . 4 5 5 9}$ \\
\hline
\end{tabular}

\section{Forecasting the Heat load Based on Neural Network}

Artificial Neural Network (ANN) is one kind of statistical learning models inspired by biological neural networks. Nowadays researchers have tried different types of neural network to forecast the short-term heat load [17]. Since many evidences indicate that the external factors strongly influence the heat load, in order to make the prediction results more accurately, the weather factors should be taken into consideration when making neural network model. According to section 2.3, the temperature, longwave radiation and humidity parameters of NWP have a higher correlation with heat load. So in this paper, these three weather parameters are considered as the inputs of ANN. The output of the ANN is the heat load forecasting result. Figure 4 shows the basic structure of ANN.

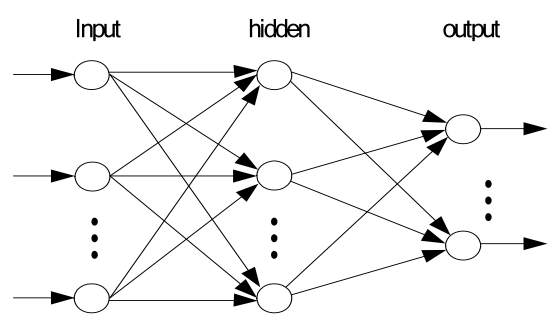

Fig.4 The basic structure of ANN 


\section{Experimental Results}

In this study, the heat load data of certain province in the north of China from October 2013 to April 2014 were used to test the performance of the proposed method. The data were arranged at intervals of 1 hour. According to the analysis of historical data, we set the training set $n=2400$. The inputs of the model are temperature, longwave radiation and humidity.

\subsection{The Forecasting results analysis}

Figure 5 indicate the heat load forecasting results of one month (Feb, 2014). From the figure, we can see that the forecasting curve fits the real heat load effectively. The Mean Absolute Percentage Error (MAPE) is $10.94 \%$ and the Root Mean Square Error (RMSE) is 0.848 . The proposed method has a considerable forecasting precision.

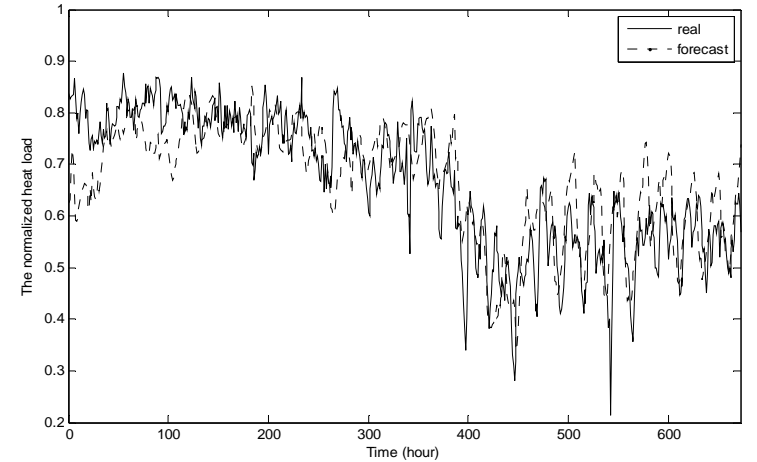

Fig.5 the heat load forecasting results (Feb, 2014)

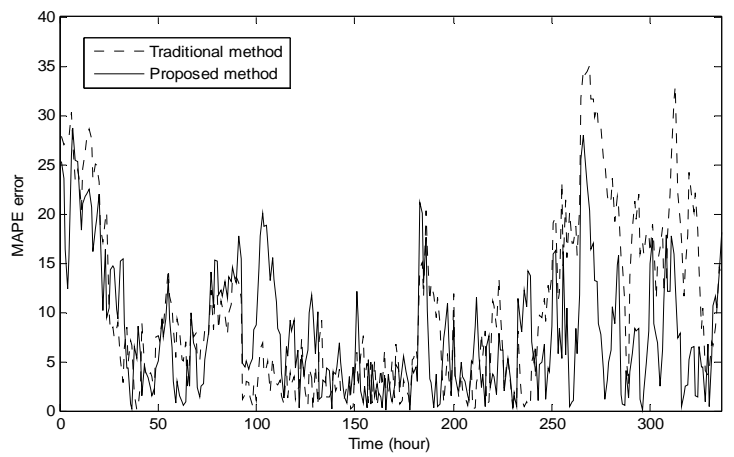

Fig. 6 the error comparison results of the two methods

\subsection{Comparison of traditional method and NWP based method}

In order to compare the effectiveness of the proposed method, the prediction using traditional weather forecast is carried out to demonstrate the advantages of NWP based method. The MAPE error of the heat load forecasting results for February 2014 is $12.60 \%$ and the RMSE error is 0.101 which are larger than the proposed NWP based method. Figure 6 shows the comparison results of the APE errors. The error curves indicate that the proposed approach has an excellent performance.

\section{Conclusion}

This paper presented an approach for heat load forecasting problem based on the NWP. After the impact factors were determined by analyzing the correlation between the weather parameters and heat load, a properly structured ANN model was designed to predict the heat load. The experimental results demonstrated that the performance of the proposed approach is better than the original weather forecast based method. The proposed method can deal with heat load forecasting with good precision.

\section{Acknowledgements}

This work was financially supported by the National Science-technology Support Plan Projects (2015BAA01B01), the National 973 Project (2012CB215101) and the state grid technology projects.

\section{References}

[1] Wang M P, Tian Q, Zhang J; Jin N N. Application of a Hybrid Algorism Combining Fuzzy Theory and Neural Network for Heating Load Forecasting. Journal of Applied Sciences. 2013, 13(10): 1911-1915.

[2] Linda Pedersena; Jacob Stangb , Rolf Ulsetha. Load prediction method for heat and electricity demand in buildings for the purpose of planning for mixed energy distribution systems. Energy and Buildings, 2008, 40(7): 1124-1134. 
[3] Wang Meiping;Tian Qi;Zhang Jiao;Jin Nana. Application of a Hybrid Algorism Combining Fuzzy Theory and Neural Network for Heating Load Forecasting. Journal of Applied Sciences, 2013, 13(10): 1911-1915.

[4] Powell, Kody M, Sriprasad, Akshay, et al. Heating, cooling, and electrical load forecasting for a large-scale district energy system. Energy, 2014(74): 877-885.

[5] Forrester J R, Wepfer W J. Formulation of a load prediction algorithm for a large commercial building, ASHRAE Trans., 1984, 90: 536-551.

[6] Amjady N.. Short-term hourly load forecasting using time-series modeling with peak load estimation capability. IEEE Trans. Power Syst. 2001, 16:798-805.

[7] Arvastson L. Stochastic modelling and operational optimization in district-heating systems. Ph. D. Thesis, Lund University, Lund Sweden, 2001.

[8] Hwang R C, Huang H C, Chen Y J, et al. Selection of influencing factors for power-load forecasting by grey relation analysis. Proceedings of the 2nd National Conference on Grey Theory and Applications (NCGTA’97), Changhua, Taiwan, 1997:109-113.

[9] Kawashima M, Dorgan C E, Mitchell J W. Optimizing system control with load prediction by neural networks for an ice-storage system.ASHRAE Trans., 1996, 102:1169-1178.

[10] Li Q, Han Y. Short-term Heat Load Forecasting of District Heating System Based on Weather. Automation and Instrumentation, 2015, 5: 5-8. (In chinese)

[11] Kelo S, Dudul S. A wavelet Elman neural network for short-term electrical load prediction under the influence of temperature. Int. J. Electr. Power Energy Syst,.2012,43:1063-1071.

[12] Milan Protić, Shahaboddin Shamshirband, et al. Forecasting of consumers heat load in district heating systems using the support vector machine with a discrete wavelet transform algorithm. Energy. 2015, 87: 343-351.

[13] Lan Bin Liu;Ai Juan Zou;Yu Fei Ma. A Method of Load Prediction in District-Heating System Based on Data Mining. Advanced Materials Research.2014, 918: 154-159

[14] Lynch, Peter. The origins of computer weather prediction and climate modeling. Journal of Computational Physics, 2008, 22(7): 3431-3444.

[15] Gregor Giebel. The State-Of-The-Art in Short-Term Prediction of Wind Power - A Literature Overview. Project ANEMOS, 2011, pp.42-45.

[16] Wang C H, Jin S L. Error features and their possible causes in simulated low-level winds by WRF at a wind farm. Wind Energy, 2014, 17(9): 1315-1325.

[17] Wang M P, Tian Q, Zhang J, Jin N. Application of a Hybrid Algorism Combining Fuzzy Theory and Neural Network for Heating Load Forecasting. Journal of Applied Sciences, 2013,13(10): 1911-1915. 